Preprint typeset in JHEP style - HYPER VERSION

\title{
Fermion Families from Two Layer Warped Extra Dimensions
}

\author{
Zhi-qiang Guo ${ }^{1}$ and Bo-Qiang $\mathrm{Ma}^{1 *}$ \\ ${ }^{1}$ School of Physics and State Key Laboratory of Nuclear Physics and Technology, Peking \\ University, Beijing 100871, China
}

\begin{abstract}
In extra dimensions, the quark and lepton mass hierarchy can be reproduced from the same order bulk mass parameters, and standard model fermion families can be generated from one generation in the high dimensional space. We try to explain the origin of the same order bulk mass parameters and address the family replication puzzle simultaneously. We show that they correlate with each other. We construct models that families are generated from extra dimensional space, and in the meantime the bulk mass parameters of same order emerge naturally. The interesting point is that the bulk mass parameters, which are in same order, correspond to the eigenvalues of a Schrödinger-like equation. We also discuss the problem existing in this approach.
\end{abstract}

Keywords: Fermion Families, Warped Extra Dimensions, Mass Hierarchy.

*Email:mabq@phy.pku.edu.cn 


\section{Contents}

1. Introduction 1

2. The Setup 3

3. Examples of metric for finite generations

4. Further Discussions 13

5. Conclusions 15

A. Explicit solutions for massive modes and zero mode: metric I

B. Numerical examples for finite generations 17

G. Property of hypergeometrical function 18

D. Solutions for zero mode and massive modes: metric II 18

E. Another metric example for orthogonality 19

F. Ricci scalar curvature for metric 20

\section{Introduction}

In recent years, extra space dimensions have played an important role in particle physics, gravity and cosmology. Many old problems get elegant solutions in extra space dimensional background with new perspectives [1], 2]. The extra space dimensions have been used to address the problems in flavor physics: why the masses of quarks and leptons distribute in a large range and have obvious hierarchy structure (the fermion mass hierarchy puzzle); why the heavier generations replicate the lightest generation with the almost same properties (the fermion family replication puzzle). There have been very interesting progress that both of them get answers in several different approaches [3, 4, 6, 6, 7].

In extra dimension, the fermion mass hierarchy has geometrical origin. It arises from the small overlap of wave functions in the extra space dimensions. The hierarchy mass structure can be reproduced with the bulk mass parameters of the same order in 5-dimension warped space (Randall-Sundrum model) successfully (for several numerical examples, see [8]). ${ }^{1}$ However, a new puzzle arises naturally: why the 5-dimension bulk mass parameters are of the same

\footnotetext{
${ }^{1}$ For definiteness, our following discussions will be based on the concise numerical example given in [9], and we hope that our discussions can apply to other examples by some modification.
} 
order, or whether we can give an explanation to the origin of the same order 5-dimension bulk mass parameters. It is easy to understand that this new puzzle correlates closely with the fermion family replication problem. One bulk mass parameter stands for one fermion family or one fermion flavor, so to explain the origin of the same order bulk mass parameter is doing the same thing to explain the family replication problem.

This new puzzle can be addressed in the approach that the three generations of standard model (SM) can be generated from one generation in the high dimensional space. This approach has been adopted in several papers [3, 5], in which the 6-dimension spacetime is reduced to 4-dimension spacetime directly. It is found that the SM families correspond to the zero modes of the high dimensional equation of motion. In these approaches, one missed the chance to give answers for the origin of the same order bulk mass parameters in 5-dimensions. In the present paper, we will adopt an alternative approach. We suggest a 6-dimension metric Ansatz of special two layer warped structure,

$$
d s^{2}=B(z)^{2}\left[A(y)^{2}\left(\eta_{\mu \nu} d x^{\mu} d x^{\nu}+d y^{2}\right)+d z^{2}\right] .
$$

As we will show below, with the help of the special structure of this two layer warped metric, we can reduce the 6 -dimension $(4+1)+1$ spacetime to 5 -dimension $4+1$ spacetime at the first step. We found that the induced 5-dimension equation of motion for fermions is similar to that analyzed in [9], while the bulk mass parameter in this induced 5-dimension equation corresponds to the eigenvalue of a 1-dimension Schrödinger-like equation, which is conformed by the fermion wave function in the sixth space dimension. Yet, because the induced 5-dimension equation of motion is similar to that analyzed in [9], we might expect that this induced 5dimension equation can be applied in this model by some modification. Hence we reduce the problem of the same order 5-dimension bulk mass parameter to the eigenvalue problem of a second order differential equation. Because the eigenvalues of a 1-dimension Schrödingerlike equation are of the same order generally, we see that the same order 5-dimension mass parameters emerge naturally. If we further reduce the 5 -dimension spacetime to 4-dimension spacetime, with the help of the model [9], the same order 5-dimension bulk mass parameters will produce the hierarchy structure of the 4-dimension physical fermion mass. By this new approach, we can address the new puzzle suggested above. We give an explanation for the origin of the same order bulk mass parameters. Because one 5-dimension bulk mass parameter corresponds to one family, we provide an answer for the origin of the families simultaneously. However, there exist some problems in this approach, we will discuss these problems in detail and suggest methods to bypass them.

According to the approach suggested above, we construct a model that the 5-dimension bulk mass parameters of the same order emerge naturally, and hence the standard model families are generated simultaneously. In fact, we can show that the 5-dimension bulk mass parameters correspond to the eigenvalues of a second order ordinary differential equation of the Sturm-Liouville type (similar to the Schrodinger equation in 1-dimension). Therefore, like the energy spectrum of Hydrogen atom, the bulk mass parameters or the families can be generated from the eigenvalues of Schrödinger-like equation with proper boundary conditions. The details will be introduced in Sec. 2. Several examples are given in Sec. 3. We give further discussions and conclusions in Sec. $⿴$ t and Sec. 5 . 


\section{The Setup}

In this section, we introduce the setup in detail. We start with the action of a bulk Dirac fermion in six dimension spacetime. The metric Ansatz for spacetime takes the form

$$
d s^{2}=B(z)^{2}\left[A(y)^{2}\left(\eta_{\mu \nu} d x^{\mu} d x^{\nu}+d y^{2}\right)+d z^{2}\right],
$$

where $\eta_{\mu \nu}=\operatorname{diag}(-1,1,1,1)$. Note that the metric has the special two layer warped structure. By this special metric structure, one can reduce the 6-dimension spacetime to 5-dimension spacetime at the first step, then further reduce the 5-dimension spacetime to 4-dimension physical spacetime, as we will introduce in detail below. We suppose that the extra dimensions both are intervals. ${ }^{2}$

The bulk action for this fermion is given by the usual form,

$$
S=\int d^{4} x d y d z \sqrt{-g}\left\{\frac{i}{2}\left[\bar{\Psi} e_{a}^{M} \Gamma^{a} D_{M} \Psi-D_{M} \bar{\Psi} e_{a}^{M} \Gamma^{a} \Psi\right]-i m \bar{\Psi} \Psi\right\},
$$

where $e_{a}^{M}$ is the sechsbien, and $D_{M}=\partial_{M}+\frac{1}{2} \omega_{M}^{a b} \Gamma_{a b}, \Gamma_{a b}=\frac{1}{4}\left[\Gamma_{a}, \Gamma_{b}\right]$ is the covariant derivative of spinor in curved spacetime. $a$ and $M=0,1,2,3,5,6$ stand for the flat spacetime indices and the curved spacetime indices respectively. The Dirac equation in 6-dimension spacetime requires $m$ to be a real number. We choose the gamma matrices representation as follows,

$$
\begin{aligned}
& \Gamma^{\mu}=\left(\begin{array}{cc}
0 & \gamma^{\mu} \\
\gamma^{\mu} & 0
\end{array}\right), \Gamma^{5}=\left(\begin{array}{cc}
0 & \gamma^{5} \\
\gamma^{5} & 0
\end{array}\right), \Gamma^{6}=\left(\begin{array}{cc}
\mathbf{1}_{4} & 0 \\
0 & -\mathbf{1}_{4}
\end{array}\right), \\
& \gamma^{0}=\left(\begin{array}{cc}
0 & -\mathbf{1}_{2} \\
\mathbf{1}_{2} & 0
\end{array}\right), \gamma^{i}=\left(\begin{array}{cc}
0 & \sigma^{i} \\
\sigma^{i} & 0
\end{array}\right), \gamma^{5}=\left(\begin{array}{cc}
\mathbf{1}_{2} & 0 \\
0 & -\mathbf{1}_{2}
\end{array}\right), i=1,2,3,
\end{aligned}
$$

where $\mu=0,1,2,3$ and $\sigma^{i}$ are the usual Pauli matrices.

By use of the metric Ansatz, the action reduces to

$$
\begin{aligned}
S & =S_{4}+S_{5}+S_{6}-\int d^{4} x d y d z \sqrt{-g} i m \bar{\Psi} \Psi \\
S_{4} & =\int d^{4} x d y d z \sqrt{-g}\left\{\frac{i}{2} B(z)^{-1} A(y)^{-1}\left[\bar{\Psi} \Gamma^{\mu} \partial_{\mu} \Psi-\partial_{\mu} \bar{\Psi} \Gamma^{\mu} \Psi\right]\right\}, \\
S_{5} & =\int d^{4} x d y d z \sqrt{-g}\left\{\frac{i}{2} B(z)^{-1} A(y)^{-1}\left[\bar{\Psi} \Gamma^{5} \partial_{5} \Psi-\partial_{5} \bar{\Psi} \Gamma^{5} \Psi\right]\right\}, \\
S_{6} & =\int d^{4} x d y d z \sqrt{-g}\left\{\frac{i}{2} B(z)^{-1}\left[\bar{\Psi} \Gamma^{6} \partial_{6} \Psi-\partial_{6} \bar{\Psi} \Gamma^{6} \Psi\right]\right\} .
\end{aligned}
$$

Varying the action with respect to $\bar{\Psi}$, we obtain the equation of motion,

$$
A(y)^{-1}\left\{\Gamma^{\mu} \partial_{\mu} \Psi+\Gamma^{5}\left(\partial_{5}+2 A^{-1} A^{\prime}\right) \Psi\right\}+\Gamma^{6}\left(\partial_{6}+\frac{5}{2} B^{-1} \dot{B}\right) \Psi-m B \Psi=0,
$$

\footnotetext{
${ }^{2}$ The conventional way in extra dimensions is to suppose the extra dimensions as orbifolds, and that the boundary conditions are determined to be the Israel's junction conditions [10], as adopted in [2]. However, as suggested in [1], the interval approach is more convenient in some cases, and it can produce more general boundary conditions. In our work, we found that it is necessary to adopt the interval approach, at least when we deal with the boundary conditions in the sixth space dimension. Of course, it is also more convenient. More about the interval approach, see [12].
} 
where $A^{\prime}=\frac{d A(y)}{d y}, \dot{B}=\frac{d B(z)}{d z}, \partial_{5}=\partial_{y}, \partial_{6}=\partial_{z}$, and the boundary term,

$$
\begin{aligned}
\delta S_{\text {bound }} & =\delta S_{5 \text { bound }}+\delta S_{6 \text { bound }} \\
\delta S_{5 \text { bound }} & =-\frac{i}{2} \int d^{4} x d z\left[\sqrt{-g} B^{-1} A^{-1} \delta \bar{\Psi} \Gamma^{5} \Psi\right]_{L}^{L^{\prime}} \\
\delta S_{6 \text { bound }} & =-\frac{i}{2} \int d^{4} x d y\left[\sqrt{-g} B^{-1} \delta \bar{\Psi} \Gamma^{6} \Psi\right]_{R}^{R^{\prime}},
\end{aligned}
$$

where we denote by $[X]_{0}^{L}$ the quantity $X_{\mid L}-X_{\mid 0}$. Denoting $\Psi=\left(\begin{array}{c}\chi_{1} \\ \chi_{2}\end{array}\right)$, where $\chi_{1}$ and $\chi_{2}$ are four-component Dirac spinors, we rewrite Eq. (2.5) as

$$
\begin{array}{r}
A(y)^{-1}\left\{\gamma^{\mu} \partial_{\mu} \chi_{2}+\gamma^{5}\left(\partial_{5}+2 A^{-1} A^{\prime}\right) \chi_{2}\right\}+\left(\partial_{6}+\frac{5}{2} B^{-1} \dot{B}\right) \chi_{1}-m B \chi_{1}=0 \\
A(y)^{-1}\left\{\gamma^{\mu} \partial_{\mu} \chi_{1}+\gamma^{5}\left(\partial_{5}+2 A^{-1} A^{\prime}\right) \chi_{1}\right\}+\left[-\left(\partial_{6}+\frac{5}{2} B^{-1} \dot{B}\right)\right] \chi_{2}-m B \chi_{2}=0 .
\end{array}
$$

Now we make the conventional Kluza-Klein (KK) decomposition. We expand $\chi_{1}$ and $\chi_{2}$ with spinor $\psi\left(x^{\mu}, y\right)$ in 5 -dimension spacetime as

$$
\chi_{1}\left(x^{\mu}, y, z\right)=\sum_{n} \widehat{F}_{n}(z) \psi_{n}\left(x^{\mu}, y\right), \chi_{2}\left(x^{\mu}, y, z\right)=\sum_{n} \widehat{G}_{n}(z) \psi_{n}\left(x^{\mu}, y\right)
$$

in which $\psi_{n}\left(x^{\mu}, y\right)$ conforms to the Dirac equation in 5-dimension spacetime,

$$
A(y)^{-1}\left\{\gamma^{\mu} \partial_{\mu} \psi_{n}\left(x^{\mu}, y\right)+\gamma^{5}\left(\partial_{5}+2 A^{-1} A^{\prime}\right) \psi_{n}\left(x^{\mu}, y\right)\right\}-\lambda_{n} \psi_{n}\left(x^{\mu}, y\right)=0
$$

As in 6-dimension spacetime, the Dirac equation in 5-dimension spacetime requires $\lambda_{n}$ to be real numbers. This can be verified by multiplying the two sides of Eq. (2.10) by $\bar{\psi}_{n}\left(x^{\mu}, y\right)$. Here we note that it is critical that $\lambda_{n}$ must be real numbers, as it will be obvious in the following discussions. With the help of Eq. (2.10), Eqs. (2.7)-(2.8) can be solved by the following Ansatz,

$$
\begin{aligned}
& \left(\frac{d}{d z}+\frac{5}{2} B^{-1} \dot{B}\right) \widehat{F}_{n}(z)-m B \widehat{F}_{n}(z)+\lambda_{n} \widehat{G}_{n}(z)=0 \\
& \left(\frac{d}{d z}+\frac{5}{2} B^{-1} \dot{B}\right) \widehat{G}_{n}(z)+m B \widehat{G}_{n}(z)-\lambda_{n} \widehat{F}_{n}(z)=0 .
\end{aligned}
$$

These equations can be simplified further by the transformations,

$$
\widehat{F}_{n}(z)=B(z)^{-\epsilon} F_{n}(z), \widehat{G}_{n}(z)=B(z)^{-\epsilon} G_{n}(z),
$$

in which $\epsilon=\frac{5}{2}$. Then we obtain the equations

$$
\begin{aligned}
& \left(\frac{d}{d z}-m B\right) F_{n}(z)+\lambda_{n} G_{n}(z)=0 \\
& \left(\frac{d}{d z}+m B\right) G_{n}(z)-\lambda_{n} F_{n}(z)=0 .
\end{aligned}
$$


For a zero mode $(\lambda=0)$, these bulk equations decouple and are easy to be solved. The solutions are given by

$$
F_{0}(z)=\frac{1}{\sqrt{l N_{0}}} \exp \left(\int_{z_{0}}^{z} m B(\zeta) d \zeta\right) \text { or } 0, G_{0}(z)=\frac{1}{\sqrt{l \widetilde{N}_{0}}} \exp \left(-\int_{z_{0}}^{z} m B(\zeta) d \zeta\right) \text { or } 0 .
$$

We introduce $l$ of the length dimension in order to make the normalization constants to be dimensionless. It will become explicit in examples in the next section. For the massive modes, we can combine the first order differential equation to obtain second order equations

$$
\begin{gathered}
\frac{d^{2}}{d z^{2}} F_{n}(z)+\left[-m \dot{B}-m^{2} B^{2}\right] F_{n}(z)+\lambda_{n}^{2} F_{n}(z)=0, \\
\frac{d^{2}}{d z^{2}} G_{n}(z)+\left[m \dot{B}-m^{2} B^{2}\right] G_{n}(z)+\lambda_{n}^{2} G_{n}(z)=0 .
\end{gathered}
$$

Rewriting them in another form, we see that they are similar to the one dimensional Schrödinger equations

$$
\begin{aligned}
-\frac{d^{2}}{d z^{2}} F_{n}(z)+V(z) F_{n}(z) & =\lambda_{n}^{2} F_{n}(z), \\
-\frac{d^{2}}{d z^{2}} G_{n}(z)+\widetilde{V}(z) G_{n}(z) & =\lambda_{n}^{2} G_{n}(z)
\end{aligned}
$$

with potentials

$$
V(z)=m \dot{B}+m^{2} B^{2}, \widetilde{V}(z)=-m \dot{B}+m^{2} B^{2}
$$

In the following discussions we will illuminate that such a setup gives answers to the puzzle we proposed in the introduction. According to the setup above, we realize an interesting fact that Eq. 2.10) is similar to the equation analyzed in the model [9] if we choose $A(y)$ to be a slice of the anti-de Sitter (AdS) metric, i.e., the RS spacetime. The differences are that there is gauge field background in the model [9], and that in that work the extra dimension is adopted to be an orbifold. However, for fermions, the equations of motion in these two cases are of almost similar features, and the gauge field background only makes the boundary conditions more involved. It is not difficult to add the gauge field background as in the model [9] to the above setup. When we further reduce the 5-dimension spacetime to 4-dimension spacetime, we can advance with the help of the model [9]. $\lambda_{n}$ in the above setup corresponds to the bulk mass parameter in 5-dimension spacetime in the model [9]. Of course, it is obvious that one bulk mass parameter corresponds to one family in 5-dimensions. Now we can understand how the same order bulk mass parameters or families are generated from extra space dimensions. The bulk mass parameters are eigenvalues of Schrödinger-like equations (2.19)-(2.20), so generally they should be of the same order. The eigenstates of equations (2.19)-(2.20), belonging to the eigenvalues $\lambda_{n}$, correspond to the generations in 5-dimensions. When we reduce further the 5-dimension spacetime to the physical 4-dimensions, these generations in 5-dimensions can produce the generations in 4-dimension spacetime. So the families in physical 4-dimension spacetime are generated simultaneously. Of course, the eigenstates should be normalizable in 
order that we can get the effective 5-dimension action after integrating out the sixth dimension. We will discuss the normalization conditions in the next section. However, a problem arises immediately from the following contradiction: On one side, the eigenvalue problem of Eqs. (2.19)-(2.20) is of the Sturm-Liouville type. The characters of the Sturm-Liouville eigenvalue problem are that the number of eigenvalues is infinite and the size of eigenvalues is non-bounded, i.e., the eigenvalue series becomes large monotonously; on the other side, as it has been illuminated obviously in the papers [9], the larger bulk mass parameters produce lighter fermions mass in 4-dimensions. Therefore, the eigenstates of Eqs. 2.19)-(2.20) produce infinite light fermion generations. However, no lighter generations are discovered by experiments so far. Hence we need another mechanics to cut off the infinite series and select only several eigenstates. The left eigenstates correspond to generations in 4-dimensions.

There also exists a problem about the zero mode (2.16). By the numerical examples in [9], the zero bulk mass parameter in 5-dimensions produces a very heavy fermion in 4-dimensions, and it is heavier than the SM generations. So it does not correspond to the physical generations. If the zero mode is permitted by the boundary conditions and the normalization conditions in our model, there would exist a generation that has not been discovered by experiment so far. However, the zero mode is more subtle in the example we will discussed in the next section. We will discuss this problem in more detail in that section.

Now we suggest several approaches to deal with the problem about the infinite eigenvalues.

(1) An immediate proposal is that one chooses a 6-dimension spacetime in which the sixth dimension is not continuous but discrete. For example, if we discrete the finite interval to be finite points, the induced Eqs. 2.19)-(2.20) will be difference equations. The number of their eigenvalues is finite naturally. There has been a similar investigation for gravity, see [13].

(2) Another bizarre proposal is that we suppose the sixth dimension to be timelike. In the Ansatz Eq. (2.1), we have chosen the sixth dimension to be spacelike. Instead we can choose it to be timelike. This leads to the metric with two time dimensions [14]. ${ }^{3}$ The alternative metric Ansatz is

$$
d s^{2}=B(z)^{2}\left[A(y)^{2}\left(\eta_{\mu \nu} d x^{\mu} d x^{\nu}+d y^{2}\right)-d z^{2}\right] .
$$

In Eq. (2.3), we let $\Gamma^{6}=\left(\begin{array}{cc}0 & -\mathbf{1}_{4} \\ \mathbf{1}_{4} & 0\end{array}\right)$, with others keeping invariant. The same procedure produces the equations,

$$
\begin{aligned}
& \frac{d^{2}}{d z^{2}} F_{n}(z)-B^{-1} \dot{B} \frac{d}{d z} F_{n}(z)+m^{2} F_{n}(z)+\left[-\lambda_{n} B^{-1} \dot{B}-\lambda_{n}^{2}\right] F_{n}(z)=0, \\
& \frac{d^{2}}{d z^{2}} G_{n}(z)-B^{-1} \dot{B} \frac{d}{d z} G_{n}(z)+m^{2} G_{n}(z)+\left[\lambda_{n} B^{-1} \dot{B}-\lambda_{n}^{2}\right] G_{n}(z)=0 .
\end{aligned}
$$

We give a simple example in which $B(z)=$ constant. The solutions are

$$
F(z)=C_{1} \exp ^{i k z}+C_{2} \exp ^{-i k z}, \quad k=\sqrt{m^{2}-\lambda^{2}} .
$$

Here we have omitted the subscripts. If we impose the boundary conditions,

$$
\left.F\right|_{0}=0,\left.\quad F\right|_{R}=0,
$$

\footnotetext{
${ }^{3}$ For extensive investigations on two-time physics, see 15
} 
then $\lambda$ must conform to

$$
k R=\sqrt{m^{2}-\lambda^{2}} R=n \pi, n=1,2,3, \cdots .
$$

As we emphasized above, $\lambda$ and $m$ must both be real numbers. Eq. (2.27) has solutions only for finite natural number. The number of the eigenvalues depends on the size of $m$, hence there are a finite number of eigenvalues.

(3) However, in the following section we will adopt another approach, i.e., we can obtain a finite number of eigenstates by choosing the metric $B(z)$ delicately. Although $B(z)$ produces infinite eigenstates generally, it is possible that some of them can produce only finite eigenstates. We will focus on this possibility in the following section, and give concrete examples for finite generations.

\section{Examples of metric for finite generations}

In this section, we will suggest metric examples which can produce finite generations. We discuss the appropriate normalization conditions for $F_{n}(z), G_{n}(z)$ at first, then we analyze examples in detail.

We begin with the action (2.4). With the help of Eqs. (2.10), (2.11) and (2.12), the action (2.4) can be rewritten as

$$
\begin{aligned}
S & =\int d^{4} x d y K_{m n}\left\{\frac{i}{2} A^{4}\left[\bar{\psi}_{m} \gamma^{5} \partial_{5} \psi_{n}-\partial_{5} \bar{\psi}_{m} \gamma^{5} \psi_{n}+\bar{\psi}_{m} \gamma^{\mu} \partial_{\mu} \psi_{n}-\partial_{\mu} \bar{\psi}_{m} \gamma^{\mu} \psi_{n}\right]\right\} \\
& -\int d^{4} x d y M_{m n} A^{5} i \bar{\psi}_{m} \psi_{n} \\
K_{m n} & =\int d z B^{5}\left(\widehat{F}_{m}^{*} \widehat{F}_{n}+\widehat{G}_{m}^{*} \widehat{G}_{n}\right)=\int d z\left(F_{m}^{*} F_{n}+G_{m}^{*} G_{n}\right) \\
M_{m n} & =\int d z B^{5}\left[\left(\widehat{F}_{m}^{*} \widehat{F}_{n}+\widehat{G}_{m}^{*} \widehat{G}_{n}\right) \frac{\lambda_{n}+\lambda_{m}^{*}}{2}\right]=\int d z\left[\left(F_{m}^{*} F_{n}+G_{m}^{*} G_{n}\right) \frac{\lambda_{n}+\lambda_{m}^{*}}{2}\right]
\end{aligned}
$$

Notice that $\bar{\Psi}=\Psi^{\dagger} \Gamma^{0}=\left(\bar{\chi}_{2}, \bar{\chi}_{1}\right)$. Eqs. (3.13.3) are satisfied for all modes, including zero modes and all massive modes.

In order to get the conventional effective 5-dimensional action

$$
\begin{aligned}
S_{5 e f f} & =\sum_{n} \int d^{4} x d y\left\{\frac{i}{2} A^{4}\left[\bar{\psi}_{n} \gamma^{5} \partial_{5} \psi_{n}-\partial_{5} \bar{\psi}_{n} \gamma^{5} \psi_{n}+\bar{\psi}_{n} \gamma^{\mu} \partial_{\mu} \psi_{n}-\partial_{\mu} \bar{\psi}_{n} \gamma^{\mu} \psi_{n}\right]\right\} \\
& -\sum_{n} \int d^{4} x d y A^{5} i \lambda_{n} \bar{\psi}_{n} \psi_{n},
\end{aligned}
$$

we consider two cases below:

Case (I): The first case is that the normalization conditions

$$
K_{m n}=\int d z\left(F_{m}^{*} F_{n}+G_{m}^{*} G_{n}\right)=\delta_{m n}
$$


are satisfied. As in the standard Sturm-Liouville case, we can convert the normalization conditions (3.5) to the boundary conditions. By Eqs. (2.17) and (2.18), we have

$$
\begin{aligned}
{\left[\lambda_{n}^{2}-\left(\lambda_{m}^{2}\right)^{*}\right] \int_{R}^{R^{\prime}} } & d z\left(F_{m}^{*} F_{n}+G_{m}^{*} G_{n}\right) \\
& =\left.\left[\left(F_{n} \frac{d}{d z} F_{m}^{*}-F_{m}^{*} \frac{d}{d z} F_{n}\right)+\left(G_{n} \frac{d}{d z} G_{m}^{*}-G_{m}^{*} \frac{d}{d z} G_{n}\right)\right]\right|_{R} ^{R^{\prime}} \\
& =\left.\left(\lambda_{n}+\lambda_{m}^{*}\right)\left(F_{m}^{*} G_{n}-G_{m}^{*} F_{n}\right)\right|_{R} ^{R^{\prime}} .
\end{aligned}
$$

In the last line, we have used the bulk equations (2.14) and (2.15) to simplify these expressions. We can also get (3.7) from Eqs. (2.14) and (2.15) directly. There are two types of concise choices to make the normalization conditions satisfied,

$$
\begin{aligned}
(a): & \left.F\right|_{R}=0,\left.F\right|_{R^{\prime}}=0 \\
\text { or } \quad(b): & \left.F\right|_{R}=\left.G\right|_{R},\left.F\right|_{R^{\prime}}=\left.G\right|_{R^{\prime}} .
\end{aligned}
$$

Then for real $\lambda_{m}$, the orthogonality is ensured by appropriate boundary conditions. In this case, we can get Eq. (3.4) from Eq. (3.1) via Eq. (3.5) in a straight way.

Case (II): The second case is that the normalization conditions Eq. (3.5) are not satisfied. In this case, $K$ and $M$ are both matrices, which means that different KK modes are mixed not just among the mass terms, but also among the kinetic terms. At the first sight, it seems that we can not get the the conventional effective 5-dimensional action Eq. (3.4). However, if $K$ is positive-definite and the number of $\mathrm{KK}$ modes is finite ${ }^{4}$, we can redefine the fermion field to get an action, which has the same form with that of Eq. (3.4). The difference is that the eigenvalues $\lambda_{n}$ are modified to different size. From Eq. (3.2) and Eq. (3.3), we know that $K$ and $M$ are both hermitian. A positive-definite hermitian matrix $K$ can be diagonalized as

$$
\begin{aligned}
K & =V^{\dagger} \Lambda V=H^{\dagger} H, \quad H=\sqrt{\Lambda} V, \\
\Lambda & =\operatorname{diag}\left(\Lambda_{1}, \Lambda_{2}, \cdots, \Lambda_{n}\right), \\
\sqrt{\Lambda} & =\operatorname{diag}\left(\sqrt{\Lambda_{1}}, \sqrt{\Lambda_{2}}, \cdots, \sqrt{\Lambda_{n}}\right) .
\end{aligned}
$$

In the above expressions, $\Lambda_{i}>0, i=1,2, \cdots, n$, as we have supposed that $K$ is positivedefinite. Redefine $\psi_{n}$ as

$$
\widetilde{\psi}_{m}=H_{m n} \psi_{n}
$$

then in the new basis $\widetilde{\psi}_{n}, M$ becomes

$$
\widetilde{M}=\left(H^{-1}\right)^{\dagger} M H^{-1}
$$

After diagonalizing $\widetilde{M}$ by $U$, we have

$$
\begin{aligned}
\widetilde{M} & =U^{\dagger} \Delta U, \\
\Delta & =\operatorname{diag}\left(\widehat{\lambda}_{1}, \widehat{\lambda}_{2}, \cdots, \widehat{\lambda}_{n}\right) .
\end{aligned}
$$

\footnotetext{
${ }^{4}$ We will give numerical examples to show that such conditions can be satisfied in Appendix B.
} 
The action (3.1) can be reduced to the form like that of action (3.4)

$$
\begin{aligned}
\widehat{S}_{5 e f f} & =\sum_{n} \int d^{4} x d y\left\{\frac{i}{2} A^{4}\left[\bar{\psi}_{n} \gamma^{5} \partial_{5} \widehat{\psi}_{n}-\partial_{5} \overline{\widehat{\psi}}_{n} \gamma^{5} \widehat{\psi}_{n}+\overline{\widehat{\psi}}_{n} \gamma^{\mu} \partial_{\mu} \widehat{\psi}_{n}-\partial_{\mu} \overline{\widehat{\psi}}_{n} \gamma^{\mu} \widehat{\psi}_{n}\right]\right\} \\
& -\sum_{n} \int d^{4} x d y A^{5} i \widehat{\lambda}_{n} \overline{\bar{\psi}}_{n} \widehat{\psi}_{n} \\
\widehat{\psi}_{m} & =U_{m n} \widetilde{\psi}_{n} .
\end{aligned}
$$

In the above, we have given the normalization conditions. The criteria are that we can integrate out the sixth dimension to get an effective 5-dimension action. Now we suggest an example that can produce finite generations. In Eqs. (2.17) and (2.18), we suppose that

$$
B(z)=s \frac{e^{\omega z}+a}{e^{\omega z}+b}, \quad s, a, b, \omega>0
$$

As in the models [2], $\omega$ can be regarded as the characteristic energy scale of the sixth dimension. We will see that it determines the size of KK modes below. The role of the dimensionless parameters $s$ and $b$ will become obvious after we give the solutions of Eqs. (2.17) and (2.18). The conditions $a, b>0$ ensure that the metric is well behaved in the interval $(-\infty, \infty)$.

Eqs. (2.17) and (2.18) can be solved by hypergeometrical functions,

$$
\begin{aligned}
F(z) & =C_{1} e^{-\mu \omega z}\left(e^{\omega z}+b\right)^{\mu-\nu} \text { hypergeom }\left(\rho-\mu+\nu, 1-\rho-\mu+\nu ; 1-2 \mu, \frac{e^{\omega z}}{e^{\omega z}+b}\right) \\
& +C_{2} e^{\mu \omega z}\left(e^{\omega z}+b\right)^{-\mu-\nu} \text { hypergeom }\left(\rho+\mu+\nu, 1-\rho+\mu+\nu ; 1+2 \mu, \frac{e^{\omega z}}{e^{\omega z}+b}\right),
\end{aligned}
$$

where $\rho=\frac{m}{\omega} s\left(1-\frac{a}{b}\right), \mu=\sqrt{\left(\frac{m}{\omega} s\right)^{2}\left(\frac{a}{b}\right)^{2}-\left(\frac{\lambda}{\omega}\right)^{2}}$, and $\nu=\sqrt{\left(\frac{m}{\omega} s\right)^{2}-\left(\frac{\lambda}{\omega}\right)^{2}} . C_{1}$ and $C_{2}$ are constants. For the sake of simplicity, we omit the subscript $n$. We only display the solution for $F(z)$ explicitly. The solution for $G(z)$ can be determined by $F(z)$ through Eq. (2.14) or by Eq. (2.18) directly. Now let us investigate this solution. For hypergeometrical function $^{5}$ Hypergeom $(\alpha, \beta ; \gamma, \xi)$, when $\operatorname{Re}(\gamma-\alpha-\beta) \leq 0$, it diverges at $\xi=1$. In the solution (3.16), we have

$$
\operatorname{Re}(\gamma-\alpha-\beta)=\operatorname{Re}(-2 \nu) \leq 0,
$$

so when $z \rightarrow \infty, \xi=\frac{e^{\omega z}}{e^{\omega z}+b} \rightarrow 1$, a singularity happens. If we choose $z$ to be a finite interval $\left[R, R^{\prime}\right]$, then the solution $(3.16)$ is well behaved in this range. Imposing the boundary conditions (3.8) or (3.9), we get infinite eigenvalues generally. However, we find that the following choice can produce a finite number of eigenvalues. Given that $z$ to be a semiinfinite interval $[R, \infty)$, then the solution $(3.16)$ develops a singularity when $z \rightarrow \infty$. This singularity makes the integral in (3.5) to be divergent. In order to make the integral to be finite, the hypergeometrical series (3.16) must be cut off to be a polynomial by the requirement $\alpha=-(n-1)$, or $\beta=-(n-1), n=1,2,3, \cdots$. In Eq. (3.16), we choose

$$
1-\rho-\mu+\nu=-(n-1), n=1,2,3, \cdots .
$$

\footnotetext{
${ }^{5}$ About the property of hypergeometrical function, see Appendix $\mathrm{C}$.
} 
Because $\lambda$ and $m$ are real numbers, as we have discussed above, Eq. (3.18) might have solutions if

$$
1 \leq n \leq\left[\sqrt{\left(\frac{a}{b}\right)^{2}-1}-\left(\frac{a}{b}-1\right)\right] \frac{m}{\omega} s .
$$

Obviously, the size of $n$ are limited by the parameters in the metric, then only finite eigenvalues are permitted. We see that it is important that $\lambda$ and $m$ are real numbers again. The conditions (3.18) are required by the boundary conditions when $z \rightarrow \infty$. This boundary condition restricts the solutions to the form (A.1)- A.3). We display these solutions in Appendix A explicitly. We see that these solutions are determined completely up to normalization constants. For these solutions, $F_{n}(z), G_{n}(z) \rightarrow x^{-\nu_{n}}$ in the symbols in Appendix A, when $z \rightarrow \infty$. The integral in (3.5) is well defined in the interval $[R, \infty)$, if $\nu_{n}>0, x>0$. Because we have chosen the range of $z$ as the interval $[R, \infty)$, we should also discuss the boundary conditions at $z=R$. We might want to follow the discussions in Case (I), that is, we require that the normalization conditions (3.5) are satisfied. Because $F_{n}(z), G_{n}(z) \rightarrow 0$ when $z \rightarrow \infty$, the normalization conditions (3.5) require that

$$
\begin{aligned}
{\left[\lambda_{n}^{2}-\left(\lambda_{m}^{2}\right)^{*}\right] \int_{R}^{R^{\prime}} } & d z\left(F_{m}^{*} F_{n}+G_{m}^{*} G_{n}\right) \\
& =-\left.\left[\left(F_{n} \frac{d}{d z} F_{m}^{*}-F_{m}^{*} \frac{d}{d z} F_{n}\right)+\left(G_{n} \frac{d}{d z} G_{m}^{*}-G_{m}^{*} \frac{d}{d z} G_{n}\right)\right]\right|_{R} \\
& =-\left.\left(\lambda_{n}+\lambda_{m}^{*}\right)\left(F_{m}^{*} G_{n}-G_{m}^{*} F_{n}\right)\right|_{R}=0 .
\end{aligned}
$$

However, it is difficult to require the solutions (A.1)- A.3 to satisfy the conditions (3.20). From (3.18), we know that $\lambda_{n}$ are determined by the parameters $\frac{m}{\omega} s, \frac{a}{b}$ and $n$. So the conditions (3.20) impose restrictions on the parameters $\frac{m}{\omega} s, \frac{a}{b}$ and the boundary parameter $R$ instead of $\lambda_{n}$. The naive numerating of parameters may mean that we can have 3 eigenstates to be orthogonal, because we have 3 parameters $\frac{m}{\omega} s, \frac{a}{b}$ and $R$. Nevertheless, such choices are difficult to be implemented and it seems less natural. It is more natural to regard the parameters $\frac{m}{\omega} s, \frac{a}{b}$ and $R$ as the input parameters, or they should be determined by unknown physics that we do not consider here. In the following discussions, we will not impose boundary conditions further at $z=R$ to determine the solutions, but we will simply give these parameters by hand to determine the solutions. In such a choice, the normalization conditions (3.5) are not satisfied. So we should change to the Case (II), that is, $K$ is matrix valued, and we try to diagonalize this matrix to get the action (3.14). Before doing that, we give numerical examples to show that only three eigenvalues are left and they are of the same order. Let $\frac{a}{b}=9 / 4, \frac{m}{\omega} s=4$. From Eq. (3.19), we know that only $n=1,2,3$ are permitted. The eigenvalues are given by

$$
\lambda_{1}=3.187 \omega(n=1), \lambda_{2}=3.833 \omega(n=2), \lambda_{3}=3.9995 \omega(n=3),
$$

which are of the same order. These massive modes together with the zero mode make $K$ and $M$ to be $4 \times 4$ matrices. In order to get the action (3.14), $K$ must be positive-definite. In Appendix B, we give numerical examples to show that $K$ is positive-definite and the modified eigenvalues $\widehat{\lambda}_{n}$ in (3.13) are still of the same order. $\widehat{\lambda}_{n}$ are given by

$$
\widehat{\lambda}_{0}=-2.69625 \omega, \widehat{\lambda}_{1}=4.00816 \omega, \widehat{\lambda}_{2}=4.44389 \omega, \widehat{\lambda}_{3}=5.26792 \omega .
$$


Here we give some interpretations for our choices of the parameters. $\frac{a}{b}=9 / 4, \frac{m}{\omega} s=4$ are chosen to ensure that only 3 massive modes are permitted. We choose $x_{0}=\frac{e^{\omega R}}{b}=30$ as the boundary value in order to ensure that the modified $\widehat{\lambda}_{n}$ are still of the same order. We found that small $x_{0}$, for example, $x_{0}=1$, makes different $\widehat{\lambda}_{n}$ to have big difference. Now we can understand the role of the parameters $s$ and $b$ in the metric (3.15). In order that we can trust our analysis, the condition $\frac{m}{\omega}<1$ should be satisfied. From the above, we see that $s$ appears in the combination $\frac{m}{\omega} s$. Then we can always keep $\frac{m}{\omega}<1$ by adjusting the value of $s$ despite of the input value of $\frac{m}{\omega} s$. While $b$ appears in the combination $\frac{e^{\omega z}}{b}$, so it is closely related to the boundary value of $z$. The role of $a$ is less obvious because it appears in a more complex way.

From the above, we notice two obvious changes: (1) The massive modes are modified to different size, but they are still of the same order; (2) The zero mode mixes with the massive modes. By this mixing, the zero mode gets mass of the same order with the massive modes. A strange point is that the zero mode gets a negative mass. However, it does not form problems in the models like [9], where only the size of the mass is relevant. In models where the sign of mass is relevant, we must reconsider whether it produces problems for our model. This new feature can supply a possibility to bypass the zero mode problem that we introduced in Sec. 2. In the above, we get 4 massive modes from the previous 3 massive modes and 1 zero mode. They can produce 4 fermion generations in 4 -dimension. This is not realistic. The above numerical example suggests us to start with 2 massive modes and 1 zero mode. If the zero mode gets mass of the same order with the massive modes through mixing as the above numerical example, we can get only 3 generations.

Before giving an example about this situation, we should discuss another mass source about the zero mode, that is, the zero mode can also get mass through coupling with a Higgs field on the brane sited at $z=R$. Here it is appropriate to introduce the brane coupling. The Wilson line phase in [9] is not well defined because the range of $z$ is noncompact. We do not suggest a concrete form for this coupling. For example, it can arise from the coupling used in [6]. Here we accept the result that the zero mode gets mass

$$
\lambda_{0}=\epsilon \omega .
$$

In the following discussions, we will consider two cases: (a) $\epsilon \rightarrow 0$, so it is negligible; (b) $\epsilon \sim 1$, so it is comparable with the massive modes. We will give numerical examples about these two cases respectively. According to the same spirit with the above example, we let $\frac{a}{b}=8 / 3, \frac{m}{\omega} s=3$ to ensure that only 2 massive modes are permitted. These massive modes are given by

$$
\lambda_{1}=2.548 \omega(n=1), \lambda_{2}=2.969 \omega(n=2) .
$$

We still choose $x_{0}=\frac{e^{\omega R}}{b}=30$, then by the same procedure with that in Appendix B, we can get the modified $\widehat{\lambda}_{n}$ as

$$
\begin{aligned}
& \widehat{\lambda}_{0}=-1.48838 \omega, \widehat{\lambda}_{1}=3.16287 \omega, \widehat{\lambda}_{2}=3.85202 \omega, \text { for } \epsilon=0 ; \\
& \widehat{\lambda}_{0}=1.71708 \omega, \widehat{\lambda}_{1}=2.59758 \omega, \widehat{\lambda}_{2}=3.19837 \omega, \text { for } \epsilon=2 .
\end{aligned}
$$

Here we only give the results. The details are similar to that in Appendix B. The zero mode gets mass of the same order with the massive modes in both cases. The difference is that the 
sign of the zero mode mass is opposite in these two cases. In both cases, with the help of the zero mode mixing with massive modes or the zero mode coupling with Higgs field, we may suggest a possibility to bypass the zero mode problem in Sec. 2. In each case, we may get just 3 generations. In models where the sign of mass is relevant, we can check which case may be realistic.

In the above example, the orthogonal conditions (3.5) are not satisfied, because we choose a special metric and a special range for $z$. This choice induces mixing between different modes. In the following, we try to construct an example, which can ensure that the orthogonal conditions (3.5) are satisfied. This example can be constructed by changing the metric (3.15) to the following form,

$$
B(z)=s \frac{e^{\omega z}-a}{e^{\omega z}-b}, \quad s, a, b, \omega>0
$$

Because $a, b>0$, this metric develops singularity ${ }^{6}$ at the point $z=\frac{\log a}{\omega}$. This may make this metric unrealistic. However, we find that it can satisfy the orthogonal conditions (3.5) just because it has such special structure. Here we let aside the problem of singularity, and focus on how it can satisfy the orthogonal conditions. The solutions can be given by hypergeometrical functions yet,

$$
\begin{aligned}
\widehat{F}(z) & =C_{1} e^{-\mu \omega z}\left(e^{\omega z}-b\right)^{\rho} \text { hypergeom }\left(\rho-\mu-\nu, \rho-\mu+\nu ; 1-2 \mu, \frac{e^{\omega z}}{b}\right) \\
& +C_{2} e^{\mu \omega z}\left(e^{\omega z}-b\right)^{\rho} \text { hypergeom }\left(\rho+\mu-\nu, \rho+\mu+\nu ; 1+2 \mu, \frac{e^{\omega z}}{b}\right),
\end{aligned}
$$

where $\rho, \mu$ and $\nu$ keep the same form with that in Eqs. (3.16). By use of the property of the hypergeometrical function, we see that if $\operatorname{Re}(1-2 \rho) \leq 0$, a singularity happens at $z=R=\frac{\log b}{\omega}$. Then if we choose the range of $z$ to be $(-\infty, R]$, like the above example, the boundary conditions at $z=R=\frac{\log b}{\omega}$ impose the conditions like (3.18). We should also make the solutions well behaved when $z \rightarrow-\infty$. These two requirements can be satisfied by the following condition

$$
\rho+\mu-\nu=-n, n=0,1,2, \cdots .
$$

For $\frac{a}{b}<1$, Eq. (3.29) has solutions and $n$ is limited by

$$
0 \leq n \leq\left[\sqrt{1-\left(\frac{a}{b}\right)^{2}}-\left(1-\frac{a}{b}\right)\right] \frac{m}{\omega} s .
$$

We give these solutions in Appendix $\mathrm{D}$ explicitly. We suppose that $\frac{m}{\omega} s=10, \frac{a}{b}=\frac{1}{2}$, in order that only 3 massive modes are permitted. Besides these massive modes, Eq. (3.29) also has a zero mode solution. These solutions are given by

$$
\lambda_{0}=0, \lambda_{1}=3.80 \omega, \lambda_{2}=4.642 \omega, \lambda_{3}=4.953 \omega .
$$

\footnotetext{
${ }^{6}$ See Appendix F.
} 
From Appendix D, we know that when $z \rightarrow-\infty$, then $x \rightarrow 0, F_{n}(z), G_{n}(z) \rightarrow 0$; while at $z=R=\frac{\log b}{\omega}, x=1$, then $F_{n}(z), G_{n}(z)=0$. So in this example, the boundary conditions (3.8) are satisfied. Then the orthogonal conditions (3.5) are ensured. There are no mixing among different modes. So in this example, the zero mode can only become massive through coupling with Higgs field. If the zero mode can get mass comparable to the massive modes, we can adjust the parameters $\frac{m}{\omega} s$ and $\frac{a}{b}$ to make that only 2 massive modes are permitted. Then we can get just 3 generations. If this zero mode gets small mass, a generation heavier than the SM generations is produced. We should check whether it is allowed by experiments. If it is objected by experiments, it will make a problem for our model.

In Appendix 国, we suggest another example, in which the orthogonal conditions (3.5) are satisfied. Like the metric (3.27), there exists a singularity in the range of $z$ we considered. In order to avoid the singularity, we may change the range of $z$. For example, for the metric (3.27), we can choose the range of $z$ to be $\left[z_{1}, R\right]$, where $z_{1}>\frac{\log a}{\omega}$. In this new range, the singularity of the metric (3.27) at $\frac{\log a}{\omega}$ is avoided. But the orthogonal conditions (3.5) will be not satisfied. We have not found a metric which satisfies the requirements: 1) it can produce finite generations; 2) it ensures that the orthogonal conditions (3.5) are satisfied, and is well behaved in the range of $z$.

\section{Further Discussions}

In this section, we compare the approach adopted in [3, 5] with our setup in Sec. 20 at first. In the approach adopted in [3, 5], the authors reduced the 6-dimension spacetime to 4-dimension spacetime directly. Distinct from this approach, we reduce two layer warped 6-dimension $(4+1)+1$ spacetime to the 5 -dimension $4+1$ spacetime which is still warped at the first step, then we reduce the 5 -dimension spacetime to the physical 4 -dimension spacetime. Their differences are the different ways that one treats the zero mode and massive modes.

(1) For the zero mode: In the approach adopted in [3, 5], the authors reduced the 6dimension spacetime to 4-dimensions directly, and got zero modes in 4-dimension spacetime, so these zero modes correspond to the standard model (SM) generations. These zero modes get mass through coupling to the Higgs field. In the present approach, we reduce the 6dimension spacetime to 5 -dimensions at first, so we get a zero mode in 5-dimension spacetime. When one further reduces the 5-dimension spacetime to the physical 4-dimension spacetime, the zero mode in 5-dimension spacetime can produce a very heavy fermion in 4-dimensions, as illuminated obviously in [9]. It is very heavy, hence it does not correspond to the SM generation. However, as we discussed in Sec. 3, the zero mode can also become massive, then it can produce SM generation if it can get large mass. But if it gets small mass, then it produces a new generation objected by experimental data. This can cause a problem for our model.

(2) For the massive modes: In the approach adopted in [3, 5], the authors got the massive modes in 4-dimension spacetime. The massive modes are heavy Kluza-Klein (KK) particles. They do not correspond to the SM fermions. However, in the present approach, we reduce the 6-dimension spacetime to 5-dimensions at first, so we get the massive modes in 5-dimension spacetime again. These massive modes are KK states in 5-dimension spacetime. When one 
reduces further the 5 -dimension spacetime to the physical 4-dimension spacetime, these massive modes in 5-dimension spacetime can produce massive fermions in 4-dimension spacetime, which are light and correspond to the SM fermions, as it is obvious in [9].

These differences provide a new chance that we can address some extra issues in the present approach: we give an explanation for the origin of the same order bulk mass parameters, and give an answer for the fermion generation puzzle in the meantime. Note that it is the special metric Ansatz (2.1) to supply such an explanation. The two layer structure of the metric enables one to reduce the 6 -dimension spacetime to 5 -dimension spacetime. The metric Ansatz for $A(y)$ can be the AdS metric,

$$
A(y)=\frac{1}{k y} .
$$

By this choice, the five dimensional Dirac equation (2.10) is similar to that analyzed in models [9].

Besides, we want to address another two issues:

(1) Whether the metric (2.1) can be the background solutions of Einstein equations? The metric Ansatz (2.1) has been analyzed in [16] in high derivative gravity with matter sources and in [17] with a negative bulk cosmological constant. Their solutions are not the metric which we suggested in Sec. 3. Here we consider a minimum coupled scalar-gravity system in order to investigate whether the metric in Sec. 3 can be realized. This is a simple and convenient way. The action is given by

$$
S=\int d^{4} x d y d z \sqrt{-g}\left\{2 M^{4} R\right\}+\int d^{4} x d y d z \sqrt{-g}\left\{\frac{1}{2} g^{M N} \nabla_{M} \phi \nabla_{N} \phi+V(\phi)\right\},
$$

in which $V(\phi)$ is the potential term for scalar field. Supposing that the metric Ansatz (2.1) and that $\phi$ only depends on $z$, we get the following equations,

$$
\begin{aligned}
4 B^{-1} B_{z z}+2 B^{-2} B_{z}^{2}+3 A^{-3} A_{y y} & =\frac{1}{4 M^{4}}\left[-B^{2}\left(\frac{1}{2} B^{-2} \phi_{z}^{2}+V(\phi)\right)\right] \\
4 B^{-1} B_{z z}+2 B^{-2} B_{z}^{2}+6 A^{-4} A_{y}^{2} & =\frac{1}{4 M^{4}}\left[-B^{2}\left(\frac{1}{2} B^{-2} \phi_{z}^{2}+V(\phi)\right)\right] \\
10 B^{-2} B_{z}^{2}+4 A^{-3} A_{y y}+2 A^{-4} A_{y}^{2} & =\frac{1}{4 M^{4}}\left[\phi_{z}^{2}-B^{2}\left(\frac{1}{2} B^{-2} \phi_{z}^{2}+V(\phi)\right)\right] \\
B^{-2} \phi_{z z}+4 B^{-3} B_{z} \phi_{z}-\frac{d V(\phi)}{d \phi} & =0
\end{aligned}
$$

in which $A_{y}=\frac{d A}{d y}, B_{z}=\frac{d B}{d z}, \phi_{z}=\frac{d \phi}{d z}$. Obviously, $A(y)$ should be of the form $A(y)=\frac{1}{k y+c}$, in which $k, c$ are constant. A minimum coupled scalar-gravity coupled system has been analyzed in [18] in five dimensions. The result is that there always exists appropriate form of $V(\phi)$ to ensure that the metric have solutions, and $V(\phi)$ and the metric can be expressed with a superpotential. The similar result applies to the above system, that is, for any $B(z)$, there exists appropriate $V(\phi)$, which makes Eqs. (4.3)-(4.6) satisfied. However, the present system is more complex, and it is difficult to express the solutions with a superpotential. Of course, in order to get the solution of the metric adopted in Sec. 3, the boundary conditions must be adopted appropriately. 
(2) Whether the examples used in Sec. 3 are acceptable physically? The metric (3.15), (3.27) and (E.1) are similar to that analyzed in [19], that is, they are both asymptotically flat. They are also both noncompact, and both have infinite volume [20]. Especially for the metric (3.27) and the metric (E.1), there is singularity [21] in the range of $z$ that we choose. So it needs further work to investigate whether they are acceptable physically. The similar problem exists for the metric (2.22), in which the sixth space dimension is timelike. As being emphasized in [22], the violations of casuality and probability give stringent restrictions on the timelike dimension. It also needs further work to investigate whether it is acceptable physically. We have not found a metric, which is finite volumed, as in the models [2], and can produce finite generations simultaneously.

\section{Conclusions}

Now we summarize the main points in our work. In this paper, we try to explain the origin of the same order bulk mass parameters, and give answers to the generation replication puzzle simultaneously. The fermion masses are of hierarchy structure in 4-dimension spacetime. It seems that it is difficult to interpret them as the eigenvalues of a Schrödinger-like equation. However, the hierarchy structure can be reproduced with the bulk mass parameters in 5-dimension spacetime. The 5-dimension mass parameters are in the same order, as have been shown in many papers [6, 7, 8]. This interesting feature supplies a chance to interpret the 5-dimension mass parameters, which are of the same order, as the the eigenvalues of a Schrödinger-like equation. Supposing that the six dimension spacetime metric has special two layer $(4+1)+1$ structure, we can reduce the 6 -dimension spacetime to 5 -dimension spacetime at the first step. We find that the bulk mass parameters are the eigenvalues of a Schrödinger-like equation. Hence the same order mass parameters emerge naturally. However, the problem is that the number of eigenvalues is infinite generally, which leads to infinite light generations. We suggest several approaches to deal with this problem. Obviously, this problem arises from the fact that in the conventional Kluza-Klein (KK) decomposition, one gets infinite KK particles generally. However, as in the example given by Madore [23], in the noncommutative geometrical background, and by the choice of the internal structure, the modification of KK theory gives rise to finite spectrum of particles. Therefore, it is possible to overcome the difficulties in our work by use of the noncommutative geometry. It will require modifying the framework in Sec. 2. We hope we can address these issues in the future.

Acknowledgement This work is partially supported by National Natural Science Foundation of China (Nos. 10721063, 10575003, 10528510), by the Key Grant Project of Chinese Ministry of Education (No. 305001), by the Research Fund for the Doctoral Program of Higher Education (China).

\section{A. Explicit solutions for massive modes and zero mode: metric I}

In this appendix, we give the solutions of Eq. (3.16) for massive modes under the conditions

(3.18) explicitly. The solutions should be well behaved in the range $[R, \infty)$. Let $x=\frac{e^{\omega z}}{b}$, the 
solutions are given by

$$
\begin{aligned}
& F_{1}(x)=\frac{\sqrt{\omega}}{\sqrt{N_{1}}} x^{-\mu_{1}}(x+1)^{\mu_{1}-\nu_{1}} \\
& F_{2}(x)=\frac{\sqrt{\omega}}{\sqrt{N_{2}}} x^{-\mu_{2}}(x+1)^{\mu_{2}-\nu_{2}}\left[1-\frac{\alpha_{1}}{\gamma_{1}} \frac{x}{x+1}\right] \\
& F_{3}(x)=\frac{\sqrt{\omega}}{\sqrt{N_{3}}} x^{-\mu_{3}}(x+1)^{\mu_{3}-\nu_{3}}\left[1-\frac{2 \alpha_{3}}{\gamma_{3}} \frac{x}{x+1}+\frac{\alpha_{3}\left(\alpha_{3}+1\right)}{\gamma_{3}\left(\gamma_{3}+1\right)}\left(\frac{x}{x+1}\right)^{2}\right],
\end{aligned}
$$

in which

$$
\begin{aligned}
\gamma_{n} & =1-2 \mu_{n}, \quad n=1,2,3, \cdots \\
\alpha_{n} & =\rho-\mu_{n}+\nu_{n}, \rho=\frac{m}{\omega} s\left(1-\frac{a}{b}\right), \\
\nu_{n} & =\sqrt{\left(\frac{m}{\omega} s\right)^{2}-\left(\frac{\lambda_{n}}{\omega}\right)^{2}} \\
\mu_{n} & =\sqrt{\left(\frac{m}{\omega} s\right)^{2}\left(\frac{a}{b}\right)^{2}-\left(\frac{\lambda_{n}}{\omega}\right)^{2}}
\end{aligned}
$$

We have dropped the hypergeometrical function after the coefficient $C_{2}$ in (3.16), because it is divergent under the conditions (3.18) when $z \rightarrow \infty$. The solutions for $G_{n}(z)$ can be gotten from (2.14) as

$$
G_{n}(x)=\frac{\omega}{\lambda_{n}}\left[\frac{m}{\omega} s \frac{x+\frac{a}{b}}{x+1} F_{n}(x)-x \frac{d}{d x} F_{n}(x)\right] .
$$

The zero mode solution is given by

$$
F_{0}(x)=0, G_{0}(x)=\frac{\sqrt{\omega}}{\sqrt{N_{0}}} x^{-\frac{m}{\omega} s \frac{a}{b}}(x+1)^{\frac{m}{\omega} s\left(\frac{a}{b}-1\right)} .
$$

When $z \rightarrow \infty, x=\frac{e^{\omega z}}{b} \rightarrow \infty$

$$
\begin{aligned}
& F_{n}(x) \rightarrow x^{-\nu_{n}}, G_{n}(x) \rightarrow \frac{\omega}{\lambda_{n}}\left(\frac{m}{\omega} s+\nu_{n}\right) x^{-\nu_{n}}, \\
& F_{0}(x)=0, G_{0}(x) \rightarrow x^{-\frac{m}{\omega} s} .
\end{aligned}
$$

They are all well behaved when $\nu_{n}>0$. In terms of $x$, the integral in Eq. (3.2) can be rewritten as

$$
K_{m n}=\int d z\left(F_{m}^{*} F_{n}+G_{m}^{*} G_{n}\right)=\frac{1}{\omega} \int \frac{d x}{x}\left(F_{m}^{*} F_{n}+G_{m}^{*} G_{n}\right)
$$

They are also well behaved in the range $[R, \infty)$ when $\nu_{n}>0$ and $x>0$. In the numerical examples we give in Sec. 3 , the conditions $\nu_{n}>0$ and $x>0$ are always satisfied. 


\section{B. Numerical examples for finite generations}

In order to get numerical results, we need to input the parameters $\frac{m}{\omega} s, \frac{a}{b}$ and $R$. From the solutions in Appendix A, we know that it is enough to input value for $x_{0}=\frac{e^{\omega R}}{b}$.

Let $\frac{m}{\omega} s=4, \frac{a}{b}=9 / 4$ as in Sec. 3 , then for massive modes, only $n=1,2,3$ are permitted. We further designate $x_{0}=\frac{e^{\omega R}}{b}=30$. We normalize the solutions for massive modes and zero mode according to $(3.5)$ as

$$
\int d z\left(F_{n}^{*} F_{n}+G_{n}^{*} G_{n}\right)=1, n=0,1,2,3
$$

These conditions determine the normalization constants. Then the matrix $K$ is determined to be

$$
K=\left(\begin{array}{cccc}
1 & 0.8797 & 0.7144 & 0.3503 \\
0.8797 & 1 & 0.9399 & 0.5173 \\
0.7144 & 0.9399 & 1 & 0.6433 \\
0.3503 & 0.5173 & 0.6433 & 1
\end{array}\right)
$$

The indices of $K$ are determined according to (3.2) as

$$
K_{m n}=\int d z\left(F_{m}^{*} F_{n}+G_{m}^{*} G_{n}\right), m, n=0,1,2,3 .
$$

$K$ can be diagonalized as

$$
\begin{aligned}
& K=V^{T} \Lambda V, \Lambda=\operatorname{diag}(3.0656,0.707478,0.211924,0.0149942), \\
& V=\left(\begin{array}{cccc}
-0.491317 & -0.555244 & -0.544655 & -0.391998 \\
-0.488159 & -0.224439 & 0.0638874 & 0.84098 \\
-0.651525 & 0.221179 & 0.626183 & -0.366729 \\
0.309555 & -0.769683 & 0.554224 & -0.0678292
\end{array}\right)
\end{aligned}
$$

where $V^{T}$ means the transpose of $V$. This example shows that $K$ is positive-definite, as we expected.

$\lambda_{n}$ is given by (3.21) in Sec. 3. $M$ is determined by (3.3) as

$$
M_{m n}=\int d z\left[\left(F_{m}^{*} F_{n}+G_{m}^{*} G_{n}\right) \frac{\lambda_{n}+\lambda_{m}^{*}}{2}\right], m, n=0,1,2,3 .
$$

Given the parameters, $M$ is determined to be

$$
M=\left(\begin{array}{cccc}
0 & 1.402 & 1.3693 & 0.7006 \\
1.402 & \frac{\sqrt{1463}}{12} & 3.2993 & 1.8589 \\
1.3693 & 3.2993 & \frac{12 \sqrt{5}}{7} & 2.5196 \\
0.7006 & 1.8589 & 2.5196 & \frac{3 \sqrt{455}}{16}
\end{array}\right)
$$


Following the procedure in Sec. 3, we can get $\widetilde{M}$ as

$$
\begin{aligned}
\widetilde{M} & =\left(\begin{array}{cccc}
2.73442 & -1.35109 & -2.28483 & 2.23697 \\
-1.35109 & 3.00477 & -1.47008 & 1.60816 \\
-2.28483 & -1.47008 & 2.19631 & 1.7851 \\
2.23697 & 1.60816 & 1.7851 & 3.08822
\end{array}\right)=U^{T} \Delta U \\
U & =\left(\begin{array}{cccc}
0.671378 & 0.216423 & -0.209452 & 0.677158 \\
-0.442926 & 0.350635 & 0.637256 & 0.524191 \\
0.256197 & -0.814717 & 0.495127 & 0.159525 \\
-0.536126 & -0.407986 & -0.552162 & 0.491155
\end{array}\right) \\
\Delta & =\operatorname{diag}(5.26792,4.44389,4.00816,-2.69625)
\end{aligned}
$$

\section{Property of hypergeometrical function}

We cite a theorem [24] about the hypergeometrical function $F(\alpha, \beta ; \gamma, \xi)$.

Theorem 2.1.2 The series $F(\alpha, \beta ; \gamma, \xi)$ with $|\xi|=1$ converges absolutely if $\operatorname{Re}(\gamma-\alpha-\beta)>$ 0 . The series converges conditionally if $\xi=e^{i \theta} \neq 1$ and $0 \geq \operatorname{Re}(\gamma-\alpha-\beta)>-1$ and the series diverges if $\operatorname{Re}(\gamma-\alpha-\beta) \leq-1$.

\section{Solutions for zero mode and massive modes: metric II}

For the metric $(\underline{3.27})$, the solutions for massive modes well behaved in the range $(-\infty, R]$ are given by

$$
\begin{aligned}
& F_{1}(x)=\frac{\sqrt{\omega}}{\sqrt{N_{1}}} x^{\mu_{1}}(1-x)^{\rho}\left[1-\frac{\beta_{1}}{\gamma_{1}} x\right], \\
& F_{2}(x)=\frac{\sqrt{\omega}}{\sqrt{N_{2}}} x^{\mu_{2}}(1-x)^{\rho}\left[1-\frac{2 \beta_{2}}{\gamma_{2}} x+\frac{\beta_{2}\left(\beta_{2}+1\right)}{\gamma_{2}\left(\gamma_{2}+1\right)} x^{2}\right],
\end{aligned}
$$

in which

$$
\begin{aligned}
\gamma_{n} & =1+2 \mu_{n}, \quad n=1,2,3, \cdots \\
\beta_{n} & =\rho+\mu_{n}+\nu_{n}, \\
\nu_{n} & =\sqrt{\left(\frac{m}{\omega} s\right)^{2}-\left(\frac{\lambda_{n}}{\omega}\right)^{2}}, \\
\mu_{n} & =\sqrt{\left(\frac{m}{\omega} s\right)^{2}\left(\frac{a}{b}\right)^{2}-\left(\frac{\lambda_{n}}{\omega}\right)^{2}} .
\end{aligned}
$$

In the above expressions, we have defined $x=\frac{e^{\omega z}}{b} . \rho$ is defined as in Sec. 3. $\lambda_{n}$ are determined by (3.29), and $n$ is limited by (3.30). We have dropped the hypergeometrical function after 
the coefficient $C_{1}$ in (3.28), because it is divergent when $z \rightarrow-\infty$. The solutions for $G_{n}(x)$ are determined by

$$
G_{n}(x)=\frac{\omega}{\lambda_{n}}\left[\frac{m}{\omega} s \frac{x-\frac{a}{b}}{x-1} F_{n}(x)-x \frac{d}{d x} F_{n}(x)\right] .
$$

The solutions (D.1)-(D.7) are well behaved when $\mu_{n}>0, \rho \geq 1$. They are satisfied in our numerical example in Sec. 3 .

We find that Eq. (3.29) also has a well behaved zero mode solution. This zero mode solution is given by

$$
F_{0}(x)=\frac{\sqrt{\omega}}{\sqrt{N_{0}}} x^{\frac{m}{\omega} s}(1-x)^{\rho}, G_{0}(x)=0 .
$$

It is consistent with what we get from (2.16).

\section{E. Another metric example for orthogonality}

In this appendix, we suggest another metric which ensures that the the orthogonal conditions (3.5) are satisfied. This metric is given by

$$
B(z)=s \frac{\omega z-a}{\omega z-b}, \quad s, a, b, \omega>0 .
$$

We suppose $a, b>0$ and $a>b$ here. The conditions $a, b<0$ and $a<b$ work well also. But the conditions $a<0, b>0$ do not work. Eqs. (2.17) and (2.18) can be solved by confluent hypergeometrical function (or Kummer's function). When $\gamma=2(1+\nu), \nu=\frac{m}{\omega} s(a-b)$ is not integer, the solutions are given by

$$
\begin{aligned}
F(z) & =C_{1} e^{-\mu(\omega z-b)}(\omega z-b)^{1+\nu} \text { hypergeom }(\alpha ; \gamma, 2 \mu(\omega z-b)) \\
& +C_{2} e^{-\mu(\omega z-b)}(\omega z-b)^{1+\nu+1-\gamma} \text { hypergeom }(\alpha+1-\gamma ; 2-\gamma, 2 \mu(\omega z-b)),
\end{aligned}
$$

in which $\mu=\sqrt{\left(\frac{m}{\omega} s\right)^{2}-\left(\frac{\lambda}{\omega}\right)^{2}}$ and $\alpha=1+\nu-\frac{m}{\omega} s \frac{\nu}{\mu}$. We choose the range of $z$ to be $[R, \infty)$, where $R=\frac{b}{\omega}$. The confluent hypergeometrical function $F(\alpha ; \gamma, \xi) \sim e^{\xi}$ when $\xi \rightarrow \infty$. In order to make the solutions well behaved when $z \rightarrow \infty$, the confluent hypergeometrical function must be cut off to be a polynomial by the requirement

$$
\alpha=1+\nu-\frac{m}{\omega} s \frac{\nu}{\mu}=-(n-1), n=1,2, \cdots .
$$

Then $\lambda_{n}$ are determined to be

$$
\lambda_{n}=\left[\frac{m}{\omega} s\left(1-\frac{\nu^{2}}{(\nu+n)^{2}}\right)^{\frac{1}{2}}\right] \omega .
$$

By (E.4), we know that

$$
\left[\frac{m}{\omega} s \frac{(2 \nu+1)^{\frac{1}{2}}}{\nu+1}\right] \omega \leq \lambda \leq\left(\frac{m}{\omega} s\right) \omega .
$$


We should also require that the solutions are well behaved at $z=R=\frac{b}{\omega}$. This condition requires the solutions further to be

$$
F_{n}(z)=\frac{\sqrt{\omega}}{\sqrt{N_{1}}} e^{-\mu(x-b)}(x-b)^{1+\nu} \operatorname{hypergeom}(-n ; \gamma, 2 \mu(x-b)) .
$$

Here we define $x=\omega z$, and $G_{n}(z)$ are determined by

$$
G_{n}(x)=\frac{\omega}{\lambda_{n}}\left[\frac{m}{\omega} s \frac{x-a}{x-b} F_{n}(x)-\frac{d}{d x} F_{n}(x)\right] .
$$

There is also a well behaved zero mode solution, which is given by

$$
F_{0}(x)=0, G_{0}(x)=\frac{\sqrt{\omega}}{\sqrt{N_{0}}} e^{-s \frac{m}{\omega} x}(x-b)^{\nu} .
$$

We have supposed $a>b$. So when $z \rightarrow \infty, x \rightarrow \infty, F_{n}(z), G_{n}(z) \rightarrow 0$; while $F_{n}(z), G_{n}(z)=0$ at $z=R, x=b$ if $\nu \geq 2$. So the orthogonal conditions (3.8) can be satisfied.

According to the analysis in [9], if we let $\lambda_{0}=m s$ to be the lightest generation of SM, there exist infinite heavier generations corresponding to $\lambda<m s$, in which $\lambda=0$ is the heaviest generation. However, there exists a problem in this case: the lighter generations approximate to be continuous, which conflicts with the experimental fact. Therefore, special boundary conditions must be adopted to remove the reductant generations. We hope that there are only finite generations left with the help of the special boundary conditions. But it seems that it is difficult to impose such boundaries naturally.

\section{F. Ricci scalar curvature for metric}

Ricci scalar curvature for metric Ansatz (2.1) is given by

$$
R=-\left[10\left(B^{-3} B_{z z}+B^{-4} B_{z}^{2}\right)+\left(8 A^{-3} A_{y y}+4 A^{-4} A_{y}^{2}\right) B^{-2}\right],
$$

in which $A_{y}=\frac{d A}{d y}, B_{z}=\frac{d B}{d z}$. From the second term in (F.1), we know that there is singularity for the metric (3.27) at $z=\frac{\log a}{\omega}$ and the metric (E.1) at $z=\frac{a}{\omega}$. At the point $z=\frac{\log b}{\omega}$ and $z=\frac{b}{\omega}$, the metric (3.27) and (E.1) are not well defined respectively, but the Ricci scalar for them are well defined. So it needs further work to determine whether they are true singularities.

\section{References}

[1] N. Arkani-Hamed, S. Dimopoulos, G. R. Dvali, Phys. Lett. B 429 (1998) 263; I. Antoniadis, N. Arkani-Hamed, S. Dimopoulos, G. R. Dvali, Phys. Lett. B 436 (1998) 257.

[2] L. Randall, R. Sundrum, Phys. Rev. Lett. 83 (1999) 4690; ibid. 83 (1999) 3370.

[3] M. V. Libanov, S. V. Troitsky, Nucl. Phys. B 599 (2001) 319; J.-M. Frère, M. V. Libanov, S. V. Troitsky, Phys. Lett. B 512 (2001) 169.

[4] B. A. Dobrescu, E. Poppitz, Phys. Rev. Lett. 87 (2001) 031801.

[5] A. Nernov, Phys. Rev. D 65 (2002) 044004; S. Aguilar, D. Singleton, Phys. Rev. D 73 (2006) 085007; M. Gogberashvili, P. Midodashvili, D. Singleton, J. High Energy Phys. 0708 (2007) 033. 
[6] T. Gherghetta, A. Pomarol, Nucl. Phys. B 586 (2000) 141; Y. Grossman, M. Neubert, Phys. Lett. B 474 (2000) 361; N. Arkani-Hamed, M. Schmaltz, Phys. Rev. D 61 (2000) 033005; D. E. Kaplan, T. M. Tait, J. High Energy Phys. 11 (2001) 051.

[7] C. Csáki, C. Grojean, J. Hubisz, Y. Shirman, J. Terning, Phys. Rev. D 70 (2004) 015012; C. Csáki, hep-ph/0510275; Y. Hosotani, S. Noda, Y. Sakamura, S. Shimasaki, Phys. Rev. D 73 (2006) 096006.

[8] Y. Hosotani, S. Noda, Y. Sakamura, S. Shimasaki, Phys. Rev. D 73 (2006) 096006; C. Csáki, C. Grojean, J. Hubisz, Y. Shirman, J. Terning, Phys. Rev. D 70 (2004) 015012; S. Chang, C. S. Kim, M. Yamaguchi, Phys. Rev. D 73 (2006) 033002.

[9] Y. Hosotani, S. Noda, Y. Sakamura, S. Shimasaki, Phys. Rev. D 73 (2006) 096006.

[10] W. Israel, Nuovo Cimento. B 44 (1966) 1; Erratum-ibid. B 48 (1967) 463.

[11] C. Csáki, hep-ph/0510275.

[12] C. Csáki, C. Grojean, J. Hubisz, Y. Shirman, J. Terning, Phys. Rev. D 70 (2004) 015012; M. Carena, J. Lykken, and M. Park, Phys. Rev. D 72 (2005) 084017.

[13] C. Deffayet, J. Mouradb, Phys. Lett. B 589 (2004) 48.

[14] J. M. Overduin, P. S. Wesson, Phys. Rep. 283 (1997) 303; M. Gogberashvili, Phys. Lett. B 484 (2000) 124; Z. Berezhiani, M. Chaichian, A. B. Kobakhidze and Z. H. Yu, Phys. Lett. B 517 (2001) 387.

[15] I. Bars, Phys. Rev. D 74 (2006) 085019.

[16] H. Collins, B. Holdom, Phys. Rev. D 64 (2001) 064003.

[17] D. Choudhury, S. SenGupta, Phys. Rev. D 76 (2007) 064030.

[18] O. DeWolfe, D. Z. Freedman, S. S. Gubser, A. Karch, Phys. Rev. D 62 (2000) 046008; C. Csáki, J. Erlich, T. J. Hollowood, Y. Shirman, Nucl. Phys. B 581 (2000) 309.

[19] R. Gregory, V. A. Rubakov, S. M. Sibiryakov, Phys. Rev. Lett. 84 (2000) 5928; C. Csáki, J. Erlich, T. J. Hollowood1, Phys. Rev. Lett. 84 (2000) 5932.

[20] G. Dvali, G. Gabadadze, M. Porrati, Phys. Lett. B 485 (2000) 208; G. Dvali, G. Gabadadze, Phys. Rev. D 63 (2001) 065007.

[21] M. Gell-Mann, B. Zwiebach, Nucl. Phys. B 260 (1985) 569; A. G. Cohen, D. B. Kaplan, Phys. Lett. B 470 (1999) 52.

[22] G. Dvali, G. Gabadadze, G. Senjanović, hep-ph/9910207; F. Yndurain, Phys. Lett. B 256 (1991) 15; M. Chaichian, A. B. Kobakhidze, Phys. Lett. B 488 (2000) 117.

[23] J. Madore, Phys. Rev. D 41 (1990) 3709.

[24] G. E. Andrews, R. Askey, R. Roy, Special Functions, Cambridge University Press, Cambridge U.K. (2000). 